\title{
ОБ ИССЛЕДОВАНИИ ПРОЦЕССОВ ГОРЕНИЯ НА ОСНОВАНИИ ПОДОБИЯ ПЕРЕНОСА ПСЕВДОВЕЩЕСТВА
}

В аэротермохимии при анализе процессов, сопровождающихся движением и взаимным реагированием массы горючего и окислителя, мы сталкиваемся с переносом материи или какого-либо ее атрибута, т. е. с явлениями переноса. В замкнутых термодинамических системах эти явления описываются известными законами сохранения массы, импульса, момента импульса и энергии. Поскольку при изучении процессов горения первостепенный интерес представляют их локальные макроскопические характеристики, то законы сохранения формулируются в дифференциальной форме применительно к элементарным объемам рассматриваемых систем. Известно, что все законы сохранения в дифференциальной форме имеют общую дивергентную форму и могут быть представлены в виде [ $\left.{ }^{1}\right]$

$$
\frac{\partial \psi}{\partial t}+\operatorname{div} j_{\psi}=I_{\psi}
$$

Следовательно, полная математическая модель процесса должна содержать в себе, как минимум, три уравнения типа (1) в виде законов сохранения массы, импульса и энергии. Поскольку при горении мы имеем дело с неоднородной средой (смесью горючего и окислителя), то уравнения переноса выводятся отдельно для каждого из компонентов горючей смеси. Их дополняет уравнение неразрывности как выражение сохранения массы горючей смеси в целом. Для замыкания системы уравнений в нее входят еще уравнение состояния газов, стехиометрическое уравнение и некоторые соотношения для определения коэффишиентов, находящихся в уравнениях переноса, - эффективного коэффициента теплопроводности, диффузии и коэффициента динамической вязкости газов. Решение такой системы уравнений при соответствующих начальных и граничных условиях дает распределение в пространстве и во времени всех параметров, определяющих процесс горения. В случае адекватности полученных распределений действительности можно было бы утверждать, что мы в состоянии создавать условия для протекания процесса в любом необходимом нам направлении, т. е. мы способны полностью управлять горением. Однако в настоящее время это еще не осуществимо. Не касаясь трудностей чисто математического характера, рассмотрим проблему определения коэффициентов переноса, которая пока не поддается решению на основании общих физических закономерностей. Эта проблема усложняется при наличии многокомпонентной горючей смеси, когда общее число коэффициентов переноса 
увеличивается. Определение всех этих коэффициентов представляет значительные трудности. Возникает вопрос, каким путем возможно хотя бы частично их преодолеть. Для отыскания соответствующих возможностей рассмотрим условия протекания горения как реакции окисления топлива.

В открытой неизолированной системе горение происходит при определенной величине концентрации окислителя, причем ход процесса предопределяется интенсивностью проникновения окислителя через пограничный слой в зону горения. В окружающей среде концентрация окислителя при этом не изменяется. В таких случаях процесс горения протекает при бесконечном избытке окислителя. При горении в закрытой изолированной системе (в камерах сгорания) определенному количеству горючего соответствует ограниченное количество окислителя, которое определяется т. н. балансовым или итоговым коэффициентом избытка окислителя. Этот коэффициент показывает, какая доля окислителя, подаваемого в камеру сгорания, остается неизрасходованной, если реакция горения в системе в целом идет до конца. Его определение на основе химического состава газовых продуктов сгорания является одной из целей известного расчета статики горения. Однако этот метод расчета рассматривает систему как целую, ее начальное и конечное состояния, и не поставляет никакой информации о промежуточных изменениях в системе.

В действительности до полного достижения конечного состояния равновесия в системах с ограниченным количеством окислителя имеют место последовательные уменьшения концентраций окислителя и горючего. C позиции статики горения такое превращение условно можно представить как стадийный процесс - переход от открытой неизолированной системы (I) к закрытой изолированной системе (II). Так, в начальный момент горения выполнены условия $c_{\mathrm{oK}}^{\boldsymbol{x}}=c_{\mathrm{o \kappa}}, \eta=0, \alpha=\infty$, которые указывают на тождественность обеих систем в исходной стадии реагирования. Далее в ходе горения $\alpha$ изменяется от $\infty$ до $\alpha_{0}, \quad \underset{c}{x}$ приобретает конечное значение $c_{\text {ок }}^{k}$ и степень выгорания $\eta$ становится равной единице (см. рисунок). Последние значения параметров соответствуют конечному состоянию системы II. Поскольку процесс выгорания макроскопически непрерывен, то в любой его период производные $d \alpha / d x$, $d c_{\text {ок }} / d x$ и $d \eta / d x$ в достаточно малых промежутках $x$ можно вычислить через отношения конечных разностей $\Delta \alpha / \Delta x, \Delta c_{\text {ок }} / \Delta x$ и $\Delta \eta / \Delta x$. Если $\Delta x$

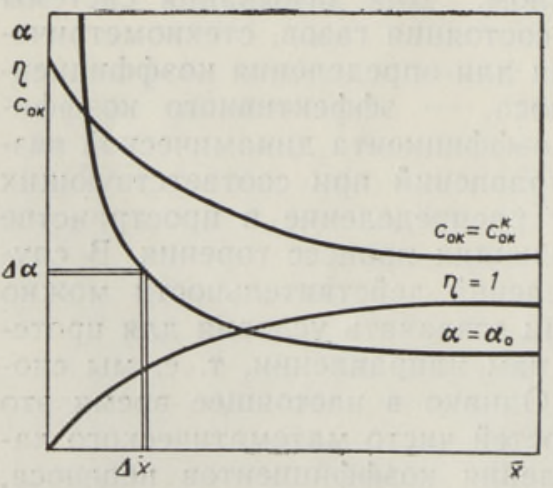

Динамика процесса горения в системе с ограниченным количеством окислителя, рассматривать как бесконечно малую величину, то соответствующий ей промежуток можно свести к элементарному объему факела. При условиях $\Delta x \approx 0$ и $\Delta \alpha \approx 0$ величина $\alpha$ оказывается инвариантной относительно элементарного объема. Следовательно, рассчитывая горение в элементарном объеме, мы можем пользоваться тем же статическим методом, что и для всей системы в целом. В этом случае устанавливаемый нами коэффициент избытка окислителя называется локальным или, по определению Г. Ф. Кнорре $\left[{ }^{2}\right]$, истинным, Он позволяет рассматри- 
вать горение от начальной стадии вплоть до конца, т. е. следить за его динамикой в пространстве и во времени. При таком подходе любая фаза горения уподобляется конечной, когда реакция достигает термодинамического равновесия. Таким образом, процесс горения можно условно рассматривать как квазистатический и соответствующие расчеты представлять как квазистатические.

В работах $\left[{ }^{3,4}\right]$ было показано, что истинный коэффициент избытка окислителя $\alpha_{\text {ист }}$ можно определить двумя, совершенно независимыми способами: во-первых, исходя из энергетического баланса или закона сохранения энергии (процесса выгорания горючего вещества) и, во-вторых, исходя из материального баланса или закона сохранения массы химических компонентов (процесса образования газовых продуктов сгорания). Однако ясно, что квазистатическое описание процесса однозначно характеризует динамику лишь в том случае, когда массоперенос между смежными элементами системы отсутствует, т. е. когда процесс одномерен. Тогда значения истинного коэффициента избытка окислителя, рассчитанные по обоим вышеуказанным способам, должны совпадать, т. е. должно осуществляться равенство $\alpha_{\text {ист }}=\alpha_{\text {ист. }}^{r}$ При наличии массообмена между элементарными объемами это равенство нарушается и мгновенная концентрация $c_{\text {ок }}^{x}$ отличается от значения, определенного из стехиометрического уравнения горения, на какую-то величину $\Delta c_{\text {ок. }}$ На основании дифференциального баланса расходования горючего и окислителя в элементарном объеме можно показать, что

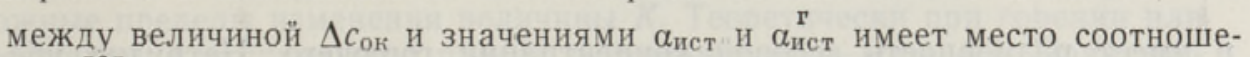
ние $\left[{ }^{3}\right]$

$$
\frac{\Delta c_{\mathrm{OK}}}{c_{\mathrm{OK}}}=\frac{1}{\alpha_{\mathrm{HCT}}}-\frac{1}{\alpha_{\mathrm{HCT}}^{\Gamma}},
$$

которое устанавливает изменение концентрации окислителя в элементарном объеме исключительно вследствие наружного массообмена и может быть рассмотрено как количественная характеристика транспорта горючего и окислителя в факеле.

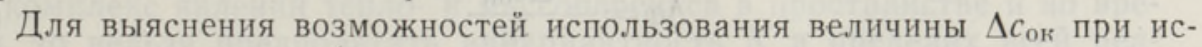
следовании горения обратимся к математическому моделированию этого процесса. Известно, что при решении задач по моделированию различных химико-технологических процессов, к которым можно отнести и процесс горения, математическое описание структуры потоков сводится обычно к описанию лишь простейших схем движения в виде моделей идеального смешения, идеального вытеснения или ячеечной модели $\left[^{5,6}\right]$. Нужно отметить, что в этих моделях принимается полное перемешивание частиц либо по всему объему реакционной камеры, либо по любому ее поперечному сечению, что, однако, находит ограниченное применение на практике. Выдвинутая в работе $\left[{ }^{7}\right]$ более общая схема течения с дискретным распределением параметров по элементарным объемам в каждом поперечном сечении потока приводит, в свою очередь, к решению сложной системы уравнений переноса массы, импульса и энергии, что было отмечено выше. В действительности же, при анализе сложных процессов оказывается возможным представление их на основании какого-то определенного и строго детерминированного явления, на которое наложено другое, дополняющее первое (иопользование принципа суперпозиции). При отсутствии напряжений сдвига и молекулярной диффузии в потоке (жесткое течение) массоперенос между отдельными элементарными объемами не имеет места. Принимая за основу такую 
идеализированную схему потока, процесс горения можно рассматривать как жесткое движение реагирующих макрообъемов горючего и окислителя, которому сопутствует диффузия какой-либо субстанции. Последняя в результате наложения на основное движение дает нам действительное явление смешения. Использование такой возможности описания процесса оказывается довольно удобным, поскольку позволяет сократить общее число коэффициентов переноса в математической модели процесса горения. В связи с этим возникает вопрос, что взять в качестве такой субстанции (псевдовещества), отсутствующей в действительности. Из определения псевдовещества ясно, что оно должно быть выражено через параметр, значение которого в одномерном процессе (жестком течении) равнялось бы нулю. При решении этого вопроса целесообразно исходить из того, что массоперенос в потоке горючего и окислителя обусловливается либо миграцией молей окислителя относительно частиц (молей) горючего, либо миграцией частиц горючего относительно молей окислителя, т. е. перемещением одной фазы относительно другой. В работе $\left[{ }^{8}\right]$ показано, что для выражения концентрации псевдовещества имеется несколько параметров. На наш взгляд, наиболее перспективен среди них тот, который учитывает разность состава горючей смеси и стехиометрического состава. Эта разность определяется соотношением $\left[{ }^{9}\right]$

$$
\varphi=x_{0}-\frac{c_{\mathrm{oK}}}{M}
$$

и имеет размерность весовой концентрации горючего. Нетрудно убедиться, что в одномерном процессе горения величина $\varphi$ остается неизменной. Параметр концентрации псевдовещества получается как разница значений $\varphi$ в реагирующем потоке горючего и окислителя при наличии массопереноса между элементарными объемами и его начальной величины (в исходной горючей смеси). Используя соответствующие соотношения из $\left[{ }^{8}\right]$, параметр концентрации псевдовещества можно выразить в виде

$$
\Delta \varphi=-\frac{\Delta c_{\mathrm{oK}}}{M}
$$

Этот параметр может оказаться весьма удобным и полезным при анализе процессов горения, поскольку его значения всегда имеют конечную величину и изменяются в небольших пределах.

Введение абстрактного понятия псевдовещества в аналитическое рассмотрение процесса горения не только упрощает математическую модель горения, но и дает возможность количественной оценки процессов массообмена на основе анализа размерностей. В частности, параметр концентрации псевдовещества фактически выражает определенную диспропорцию между протеканием процессов смешения и выгорания в горящей среде, известную «асинхронизацию» между этими процессами. Поскольку $\Delta \varphi$ имеет размерность плотности массы, то отношение этой величины к исходной весовой концентрации горючего вещества в элементарном объеме в начале процесса является мерой названной диспропорции и может служить критерием подобия, выражаюшим неравномерность расходования окислителя в процессе горения. Обозначим этот критерий через $K$ и представим его в виде

$$
K=\frac{\Delta \varphi}{\varkappa_{0}}=-\alpha_{0} \cdot \Delta \bar{c}_{\mathrm{oK}},
$$




$$
\Delta \bar{c}_{\mathrm{OK}}=\frac{\Delta c_{\mathrm{OK}}}{c_{\mathrm{oK}}} .
$$

Для определения $K$ непосредственно из данных по выгоранию горючего удобно использовать следующие формулы расчета:

а) при горении твердого диспергированного топлива

$$
K=\frac{\alpha_{0}}{\alpha_{\text {ист }}^{r}}-\eta,
$$

где $\eta=1-q_{4}$.

б) при горении жидкого топлива и газа

$$
K=\eta \frac{\alpha_{0}}{a_{\text {ист }}^{r}}-\eta,
$$

где $\eta=1-q_{3}$.

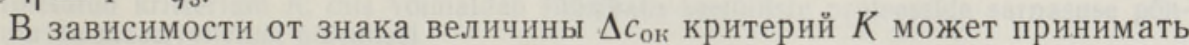
либо положительные, либо отрицательные значения. Согласно выражению (4), положительные значения означают приращение псевдовешества в элементарном объеме (при режиме газификации топлива), 'а отрицательные, соответственно, его убывание (при разбавлении горючей смеси окислителем). Соотношения (6) и (7) позволяют оценить возможные пределы изменения величины $K$. Теоретически при горении или газификации топлива этот предел составляет от -1 до +1 . Практически же при горении в открытой неизолированной системе $K$ изменяется от 0 до - 1 , а в закрытой изолированной системе при полной герметичности камеры сгорания возможны лишь ее локальные отклонения в любую сторону от нуля, что обусловливается исключительно перераспределением концентраций горючего и окислителя.

Если значения $K$ оказываются инвариантными в каких-то реагирующих системах, то это свидетельствует о подобии процессов массообмена в этих системах. Поскольку процесс горения представляет собой сложный комплекс явлений тепло- и массопереноса в пространстве и во времени, то нельзя предполагать инвариантность $K$ в пределах всей реагирующей системы, а необходимо найти его распределение. Согласно общим принципам моделирования физико-химических процессов, разработанным Г. К. Дьяконовым $\left[{ }^{10} 1\right.$, следует отыскать возможности связывания критерия $K$ с какими-либо другими, определяющими критериями процесса, которые составлены из переменных, входяших в условия однозначности. По существу $K-$ критерий определяемый, поскольку содержащаяся в нем величина $\Delta c_{\text {оқ }}$ отражает кинетические и транспортные свойства системы. Физический смысл критерия $K$ заключается в том, что он является мерой перераспределения (переноса) в горяшей среле массы горючего и окислителя относительно их исходного распределения (концентрации) в горючей смеси. Задачи исследования состоят. таким образом, в нахождении критериального соотношения для $K$. которое охарактеризовало бы подобие процесса горения с точки зрения массопереноса внутри факела. Пока мы еше не в состоянии точно определить, какие безразмерные величины следует принять в качестве аргументов в таком критериальном соотношении. Вероятно, прежде всего в число этих критериев должны вхолить безразмерные координаты пространства, позволяюшие искать геометрическое подобие систем. Для установления гидродинамического, теплового и физико-химического полобия в критериальное соотношение должны быть включены и критерии $\operatorname{Re}$ (или 
$\mathrm{Gr}, \mathrm{Fr}), \operatorname{Pe}, \mathrm{Pe}_{д}, \operatorname{Pr}, \mathrm{Pr}_{д}, \operatorname{Arr}$, критерий безразмерной концентрации частиц топлива, критерий пропорциональности полей температур и концентраций горючего вещества и др. Следовательно, искомое нами соотношение между величиной $K$ и определяющими критериями процесса в общем выражении можно представить функциональной зависимостью вида

$$
K=\Phi\left(\frac{x}{l} ; \frac{y}{l} ; \operatorname{Re} ; \operatorname{Pe} ; \operatorname{Pr} ; \operatorname{Pe}_{д} ; \operatorname{Pr}_{д} ; \operatorname{Arr} ; \frac{\chi}{\chi_{0}} \ldots\right)
$$

При выделении из общего количества явлений основных Факторов и определяющих их величин число критериев-аргументов в соотношении (8) может значительно сокращаться. Если бы вывод такого критериального соотношения был осуществим, то это дало бы новое основание для количественного анализа процессов, имеющих место в горящем факеле, а вместе с тем, способствовало бы в известной мере и дальнейшему развитию математического моделирования процессов горения.

\section{О бозн ачен ия}

$\psi$ - субстанция материн;

$t$ - время;

$j_{\psi}-$ поток субстанции $\psi$ через единицу поверхности в единицу времени;

$I_{\psi}-$ плотность источников (стоков) субстанции $\psi ;$

$c_{\text {ок }}, \underset{\boldsymbol{x}}{\boldsymbol{x}}$ ок - весовые концентрации окислителя в исходной горючей смеси и в одномерном факеле;

$\Delta c_{\text {ок }}$ - разность между действительной концентрацией окислителя и мнимой концентрацией в случае одномерного выгорания горючего;

$\eta$ - степень выпорания;

$\alpha_{\text {ист }}$ - истинный коэффициент избытка окислителя, определенный по выгоранию горючего;

$\alpha_{\text {nст }}^{\mathbf{r}}-$ истинный коэффициент избытка окислителя, определенный по химическому составу газовых продуктов сгорания;

$\alpha_{0}$ - итоговый (балансовый) коэффициент избытка окислителя в исходной горючей смеси;

$x_{0}$ - концентрация горючего вещества в исходной смеси;

$M$ - стехиометрический коэффициент;

$q_{4}$ - механический недожог топлива;

$q_{3}$ - химический недожог топлива;

$\chi, \chi_{0}$ - весовые концентрации диспергированных частиц топлива в исходной горючей смеси и в горящем факеле, приведенные к первоначалыной массе.

\section{ЛИТЕРА Т У Р А}

1. Колесников П. М., Энергоперенос в неоднородных средах, Минск, 1974.

2. Кнор е Г. Ф.. Арефьев К. М.. Блох А. Г., Нахапетян Е. А.. П л е в И. И., Ш т й н берг В. Б., Теория топочных процессов, М.-Л., 1966.

3. Прес с В., Изв. АН ЭССР, Физ. Матем., 22, 312 (1973).

4. П р ес с В., Изв. АН ЭССР, Физ. Матем., 24, 237 (1975).

5. К а фа ров В. В., Основы массопередачи, М., 1972.

6. З а кгей м А. Ю., Введение в моделирование хнмико-технологических процессов, M., 1973.

7. П рес с В., Изв. АН ЭССР. Физ. Матем., 22, 401 (1973).

8. П р ес с В., Изв. АН ЭССР, Физ. Матем., 24, 215 (1975).

9. Госмен А. Д., Пан В. М., Ранчел А. К., Сполдинг Д. Б., Вольф. 
ш те й н М., Численные методы исследования течений вязкой жидкости, M., 1972.

10. Дьяконов Г. К., Вопросы теории подобия в области физико-химических процессов, М., 1956.

Ннститут термофизики и электрофизики Академии наук Эстонской ССР

Поступила в редакцию 2/III 1976

\section{PRESS}

\section{POLEMISPROTSESSIDE UURIMISEST PSEUDOAINE ULEKANDE SARNASUSE ALUSEL}

Kirjeldatakse võimalust uurida põlemisprotsesse kvaasistaatilise käsitluse kaudu, põlevaine ja hapendaja kulu diferentsiaalbilansi alusel. Esitatakse meetod põlevaine ja hapendaja ülekande kvantitatiivseks hindamiseks leegis. Dimensioonanalüüsist lähtudes on tuletatud kriteerium $K$, mis võimaldab füüsikalis-keemiliste protsesside sarnasuse pōhjal uurida massivahetust põlemisel.

\section{PRESS}

\section{ON THE INVESTIGATION OF BURNING PROCESSES ON THE GROUND OF SIMILARITY OF PSEUDOSUBSTANCE TRANSFER}

In the paper the possibilities of the investigation of burning processes at their quasistatic conception by the use of differential balance between the consumption of fuel and oxidant are described. A method for quantitative estimation of fuel and oxidant transport in flame is presented. Proceeding from dimensional analysis, the criterion $K$ is derived, which enables to study the mass transfer phenomena at burning on the ground of the similarity theory of physico-chemical processes. 